\title{
An Alternative Approach to Existence Monism: An Interpretation of Truisms Using Linguistic Ontology and the One as Semantic Glue
}

\author{
Masahiro TAKATORI*
}

\begin{abstract}
Existence monism (EM) is a metaphysical view asserting the existence of only one concrete object. EM is well known for its radicalness, and encounters difficulty in terms of its prima facie inconsistency with truisms. This paper aims to propose an alternative (and somewhat easy) way to overcome this difficulty and indicate another means by which the possibility of EM can be defended. I will present a package of theses that are intended to be combined with EM, which I call Linguistic Ontology with the One as Semantic Glue (LOOSG). I will show that this package (in combination with EM) provides a systematic explanation as to why truisms hold while only one concrete object actually exists. In other words, I will argue that if an existence monist embraces LOOSG, the desired explanation for truisms is then available to her. In addition, it will also be noted that LOOSG has a theoretical virtue, in that it only presupposes the framework of standard semantics. Based on these discussions, I offer LOOSG as a viable option for existence monism.
\end{abstract}

Key words: existence monism, term-model, referential magnetism, linguistic idealism

\section{Introduction}

Existence monism (EM) is the metaphysical view that there is only one concrete object in reality. EM is a genuinely radical view and immediately confronts a serious difficulty - the prima facie inconsistency with truisms. That is, EM looks obviously incompatible with ordinary truths and/or the main body of scientific knowledge, such

\footnotetext{
* Keio University

e-mail: masahiro_takatori@a3.keio.jp

I would like to deeply thank Takeshi Akiba and Ikuro Suzuki for giving me a great deal of advice as well as encouraging me to finish the paper. I am also very grateful to two anonymous referees of this special issue for providing many valuable comments to improve the manuscript.
} 
as "there are two chairs in this room" or "there are eight planets in the solar system." Although EM has often been taken up seriously throughout the history of philosophy, most contemporary metaphysicians seemingly regard this view as implausible, and sometimes even unintelligible. ${ }^{1}$ If one wants to defend EM properly, one should at least resolve the prima facie inconsistency with truisms - in other words, one should at least provide an explanation as to why truisms (apparently) hold while only one concrete object actually exists.

The present paper aims to defend existence monism modestly and in a restricted manner. Herein, I propose an alternative and easy means of overcoming the major difficulty confronted by EM stated above and indicate another route for defending a possibility of EM. To this end, I present a package of theses intended for combination with EM, which I call Linguistic Ontology with the One as Semantic Glue (LOOSG). I will demonstrate that when it is combined with this package, EM can provide a systematic explanation for truisms' actually being true. Stated differently, I argue that if an existence monist embraces LOOSG, the desired explanation for truisms is then available to her. Furthermore, from a technical perspective, when EM is combined with LOOSG, it has a theoretical virtue, in that it presupposes the framework of standard model-theoretical semantics with which most metaphysicians are familiar. As such, I conclude that LOOSG is at least a viable option for existence monism.

This paper is organized as follows. In Section 1, I formulate the claim of EM in a standard manner (1.1) and make a brief remark on the major motivations for it (1.2). In Section 2, I again articulate the problem of inconsistency with truisms (2.1) and provide critical commentary on two representative approaches that aim to address this problem (2.2). Section 3 comprises the main portion of this paper. First, I explain how the notion of truth can be characterized within the framework of standard model-theoretical semantics (3.1). Second, I focus on the existence of what is called a term-model for any consistent theory (3.2). I then introduce the package of theses, which I reference as LOOSG. I comment on each thesis of LOOSG and demonstrate that given this package, we can derive the truth of truisms (3.3). In Section 4, I respond to possible objections to LOOSG, which can be easily expected.

\footnotetext{
${ }^{1}$ Historically, Parmenides (in On Nature), Spinoza (in his Ethics) and Bradley (Bradley (1994)) are known as prominent supporters of EM (though there are controversies over interpreting these philosophers as existence monists). However, I do not pursue historical issues here, nor do I claim that my proposal here would have been accepted by these past thinkers. Among contemporary metaphysicians, Terence Horgan and Matjaž Potrč are notable and exceptional advocates of EM (Horgan \& Potrč (2008)). I will make some critical remarks on their position in Section 2.2 .
} 


\section{The Gist of the Existence Monism}

\subsection{Formulation of Existence Monism}

Following Jonathan Schaffer's exposition (cf. Schaffer (2018)), we can formulate the claim of existence monism as follows ( ' $C$ ' is a predicate which stands for being a concrete object):

EM $\exists x(C x \& \forall y(C y \rightarrow y=x))$

(Hereafter, the abbreviation 'EM' will be used in two ways: one is for existence monism as a metaphysical position, and the other is the name for the thesis formulated above. This is to economize the number of abbreviations and using 'EM' in both ways will not cause any serious confusion.)

Three points should be noted here. First, EM is a thesis regarding concrete objects, such as middle-sized material objects, persons, various objects supposed in the field of natural sciences, among others. ${ }^{2}$ The existence monist must deny that there are two or more such entities. However, EM says nothing about abstract objects. Thus, she has no trouble with admitting that there are many abstract entities such as properties or various abstract types. In particular, it is noteworthy that she can accept the existence of various mathematical objects and linguistic types (this point concerns the main idea of my proposal in Section 3).

Second, EM says little about the nature of a concrete object, the existence of which is claimed by this thesis. Regarding the ontological status of this concretum, multiple options are open to a defender of EM. When existence monism is discussed in contemporary debates, there is a tendency to identify this concretum with the whole cosmos (e.g. Horgan \& Potrč (2008)). However, we shall remain neutral here and use the boldface and capitalized term 'the One' as the constant to designate the concretum in question.

Third, EM is sharply distinguished from the claim of priority monism: the thesis that there exists only one ontologically basic (or fundamental) concrete object. As Schaffer emphasizes in various places (e.g., Schaffer (2007), (2008), (2018)), priority monism is a far weaker and likely less radical doctrine than EM. Although the renaissance of monism in contemporary metaphysics is more focused on priority monism (cf. Schaffer (2008), (2018), Goff (2012)), this paper focuses on existence monism.

\subsection{Motivations for Existence Monism}

Although providing preferential support for existence monism is far beyond the

\footnotetext{
${ }^{2}$ I assume that the notion of concrete object is sufficiently understood and do not discuss its exact characterization in this paper. In fact, the details on this point (for example, the discussion as to whether spacetime points should be classified as concrete objects) do not affect the main portion of my argument in Sections 3 and 4 .
} 
scope of this paper, it is helpful to give a brief overview of some motivations for EM here.

\section{Ontological Simplicity}

As a metaphysical position, EM is obviously characterized by the virtue of ontological simplicity or parsimony. That is, according to EM, no concrete object exists but the One. Therefore, embracing EM yields a significantly more parsimonious ontology than other metaphysical views, which posit various concrete things.

\section{Resolution of the Special Composition Question}

Under what conditions do some (concrete) parts compose a whole object? This problem is known as the Special Composition Question (SCQ) in contemporary metaphysics. The difficulty in dealing with this problem is that most methods for specifying such conditions seemingly involve some amount of undesirable arbitrariness (cf. Horgan \& Potrč (2008), Ch.2 for more details). Meanwhile, once we endorse EM, SCQ is resolved in a rather trivial manner; according to EM, there are no concrete parts that potentially compose another object after all. Although this resolution seems somewhat radical, EM has a certain level of problem-solving ability on this point.

\section{Resolution of the Problem of Vague Objects}

Horgan and Potrč pose the following dilemma: (1) commonsensical concrete objects involve ontological vagueness, but (2) ontological vagueness is impossible. (cf. (2008), Ch.2; see also Schaffer (2018), Section 2.2.3. However, there are also some unignorable objections, e.g., Lowe (2012).) A specific version of EM is then considered as a viable means of circumnavigating this dilemma; no concrete object exists but the whole cosmos as the One, and the cosmos is never a commonsensical object with ontological vagueness (Horgan \& Potrč (2008), Ch.4).

I do not claim that these points make existence monism a more promising candidate than other familiar and nonradical metaphysical positions. However, these points do prompt some motivation for pursuing a possible defense of existence monism.

\section{Major Problem for Existence Monism}

\subsection{Inconsistency with Truisms}

As stated in the Introduction, existence monism is prima facie inconsistent with our ordinary truths and/or scientific knowledge, which have been firmly established. For example, it seems obvious that there are two chairs in this room. Alternatively, astronomical research has seemingly firmly established the existence of two moons 
around Mars. Any of such truths apparently implies the existence of at least two concrete objects - which constitutes the precise denial of EM. Hereafter, I shall use the term 'truisms' for the totality of ordinary truisms and scientific truths, and will also use ' $T$ ' as the signifier of this totality. Thus, in short, EM faces the difficulty of prima facie inconsistency with $T$.

If one wants to defend EM properly, resolving the prima facie inconsistency with $T$ is the primary task. This task can be rephrased as that of providing some explanation as to why truisms apparently hold while there actually exists only one concrete object. The remainder of this paper is dedicated to exploring how such an explanation may be given.

Here, it should also be noted that the explanation for truisms is expected to be systematic. In other words, the explanation in question is required to preserve the logical/inference relations among the members of $T$ for as long as possible. A candidate for the desired explanation can be evaluated by whether said candidate satisfies this requirement of systematicity.

\subsection{Two Major Strategies}

In this subsection, I provide critical commentary on two major approaches that may enable proponents of existence monism to address the problem of inconsistency with $T$.

\section{Paraphrase Strategy}

A relatively well-known approach to this problem is paraphrasing. For clarity, let us for the moment adopt a specific version of EM, according to which the One is identified with the cosmos (the whole world). The existence monist then claims that truisms apparently talking about concretums are paraphrased into the sentences that only talk about the cosmos and its modes. For example, given that the sentence 'There are two chairs in this room' is a member of $T$, this sentence is paraphrased as 'the cosmos is chairish here and there.' Here, the latter sentence is no longer inconsistent with the claim made by EM. An advocate of this approach claims that this paraphrasing technique - in which ordinary statements are converted into cosmos-mode sentences - works for other sentences as well.

As suggested above, the primary task of paraphrase strategy is to find a generalized and systematic way of paraphrasing into this sort of cosmos-mode language. Unfortunately, no such paraphrasing method has been established or widely accepted in contemporary debates. ${ }^{3}$ In addition, the following point should also be noted: according to the paraphrase strategy, any constituent of truisms is not true in the

\footnotetext{
${ }^{3}$ There are some controversies on this point. cf. Sider (2007), (2008); Cornell (2013); see also Schaffer (2018), Section 2.2.1 as a summary.
} 
strict sense. That which is true in the strict sense is the paraphrasing sentence in the cosmos-mode language that corresponds to the original sentence of $T$. This point may also be considered as a disadvantage (cf. Horgan \& Potrč (2008), Ch.5.7.2).

I do not claim that paraphrase strategy has fatal problems, nor do I claim that this strategy is unattractive. ${ }^{4}$ However, given the above remarks, we should say that this strategy still remains unsatisfactory in view of whether it provides a sufficient explanation for truisms.

\section{Contextual Semantics by Horgan \& Potrč}

Today, Terence Horgan and Matjaž Potrč are exceptional advocates of existence monism and advance their own version of it (Horgan \& Potrč (2008)). Their main idea consists of revising the semantics; instead of adopting standard (model-theoretical) semantics, they offer an alternative semantical framework that they call contextual semantics. For example, in this framework, the statement 'There are two chairs in this room' is true even if chairs do not exist or there are no ordinary objects at all. Roughly speaking, in the framework of contextual semantics, the notion of truth is characterized as an indirect correspondence between language and the world. Through this indirect correspondence, the truth of 'There are two chairs in this room' is explained as the cosmos (the "blobject," in their terms) having some distributional properties in the appropriate arrangement. Similarly, any statement included in truisms can be interpreted as some attribution of properties to the cosmos, and its truth does not need any concrete object other than that of the cosmos (according to their argument).

In my view, the idea of focusing on matters at the semantic level is significant. In this regard, their approach is aligned with that of my own proposal, which is discussed in the next section. Nevertheless, their framework has a serious flaw: lacking details crucially. As some critics have raised complaints on this point (Schaffer (2012), Ch.3.2.2; also see Korman (2008)), they simply discuss their strategy's direction and never address the task of building a semantic theory in their framework. For example, they never offer a general or systematic way of specifying a truth-condition for each sentence. On the whole, contextual semantics remains an obscure program and in their own words, comprises a mere "skeleton." Although they attempt to vindicate their attitude on this point by appealing to methodological arguments (Ch.6), their discussion is far from persuasive. In summary, as far as it relates to contextual semantics, their strategy does not provide the desired explanation.

\footnotetext{
${ }^{4}$ In fact, my proposal in Section 3 (the package of LOOSG) can be regarded as a sort of paraphrase strategy. In one regard, the explanation provided by LOOSG amounts to paraphrasing into a metalanguage talking about only object-language expressions, mathematical objects, the One and their (semantic) relations.
} 


\section{Another Strategy for Existence Monism: Linguistic Ontology with the One as Semantic Glue}

I will now consider another neglected but simple approach to providing an explanation for truisms. In 3.1, I will explain how the notion of truth can be characterized in the framework of standard model-theoretical semantics. In 3.2, I will note the existence of a term-model for any consistent first-order theory. In 3.3, I will then propose the package of theses comprising Linguistic Ontology with the One as Semantic Glue (LOOSG), comment on each thesis therein, and demonstrate that LOOSG yields the desired explanation sought in the previous section. ${ }^{5}$

\subsection{Truth in Model-Theoretical Semantics}

Hereafter, I assume the following: (1) $T$ is a theory formalized in some classical first-order language and (2) $T$ is consistent. These assumptions are reasonable here; it is frequently assumed that the totality of truisms are formalized in standard first-order predicate logic, thus (1) is reasonable enough here. (2) is also sufficiently plausible because $T$ is the set of truisms. In addition, I presuppose the framework of standard, textbook-style model-theoretical semantics to interpret $T$. Here, I emphasize that this presupposition is entirely consistent with the claim of EM. It should be noted that the framework of model-theoretical semantics itself posits only linguistic types of object language and mathematical objects (pure mathematical objects and mathematical constructs from linguistic expressions) $;^{6}$ this framework itself does not require any concrete object. The framework of model theoretical semantics says little regarding the kind of objects that are included in the domain of a given model - in particular, this does not require a model to contain concretums in its domain.

Now, when we handle the notion of truth in the framework of model-theoretical semantics, we should distinguish two notions: truth in a model (or truth relative to a model) and absolute truth (or truth simpliciter). The former is, namely, satisfaction relation between a model and a sentence/theory. The latter reflects the more

${ }^{5}$ Although the contexts are distinct, it is noteworthy that my proposal is heavily influenced by Robbie Williams's argument regarding the existence of a Pythagorean model for the best theory (Williams (2007), Sections 3 and 4). In particular, the idea of focusing on the existence of a term-model for the best theory plays a key role in both discussions. However, his own point focuses on disclosing a problem regarding Lewis's explication of the notion of eligibility.

${ }^{6}$ In fact, model theory as a branch of pure logic does not need linguistic objects to be distinguished from mathematical objects (by the well-known technique of Gödel numbering, linguistic objects are easily identified with some natural numbers). However, model-theoretical semantics, as a means of interpreting actual language that we (or metaphysicians) use, still requires genuine linguistic objects. 
ordinary usage of the truth-predicate and corresponds to the usual notion of truth. ${ }^{7}$

How are these two notions related? It is easily observed that truth in a model itself cannot be equal to absolute truth. For any consistent sentence/theory, we can easily construct numerous models, so many false sentences/theories have a model. To characterize absolute truth by truth in a model, we have to pick up some privileged model - absolute truth is defined as truth in this privileged model. Traditionally, what is called an intended model has been supposed to be a good candidate of this kind of privileged model. So, do we have to specify what is an intended model of a given theory here? In fact, to articulate the privilege of this kind of model, we do not need to examine the details of an intended model itself; rather, we should focus on its function. That is, we can extract the function of an "intended model" as follows: to determine the reference/interpretation of a given theory. In other words, the privileged model in question can be characterized as the model at which the referents (or interpretation) of the object language expressions are determined. We can also notice that, in the context of discussing (in)determinacy of reference, metaphysical realists seemingly regard this role as what an intended model plays de facto. ${ }^{8}$ Henceforth, I use the term "referentially privileged model" for this kind of privileged model characterized as such. ${ }^{9}$

Once the notion of referentially privileged model is introduced, the following question arises: what makes a given model referentially privileged? In other words, what are the grounds for a given model's being referentially privileged? This is a problem concerning the very basis of metaphysical realism and some proposals have been made to provide such grounds. For example, the idea of an eligible model by David Lewis is well known among such proposals (cf. Lewis (1983), (1984); see also Sider (2011), Ch.3). According to this proposal, a given model's being referentially privileged is grounded (or explained) by its being eligible, and the notion of eligibility is analyzed through using the notion of naturalness. However, even if the idea of eligible model is the most popular among mainstream metaphysicians, this does not mean that this idea is the only option for establishing the grounds for a given model

${ }^{7}$ Certainly, such a view is very simplified and ignores many parameters in natural language. However, this kind of simplification seems insubstantial and is therefore allowable for the present purpose.

8 This point can be observed from many literatures of the topic (e.g. Lewis (1983), (1984), Williams (2007)).

${ }^{9}$ It should be emphasized that referentially privileged models characterized in this way do not need to coincide with what are called intended models. As stated, in characterizing a referentially privileged model, we have extracted the function of a model usually called an "intended model", and fulfilling this function does not mean being the same as the latter. In fact, my proposal in 3.3 amounts to asserting that the referentially privileged model of $T$ is not $T$ 's intended model in its usual sense. I will mention this point again in Section 4. 
to be referentially privileged. Another candidate can be considered to provide such grounds. My proposal will address this point in 3.3.

Anyway, on the basis of the considerations above, we can embrace the following biconditional ( $m$ is a variable for models):

DT $T$ is true (simpliciter) if and only if there exists $m$ such that $m$ satisfies $T \& m$ is referentially privileged.

\subsection{Term-Model}

I will now focus on the notion of term-model and the metalogical theorem that for any consistent classical first-order theory there exists a term-model. This is just an elementary theorem in metalogic and I will therefore simply sketch the outline using a very simple, toy example. ${ }^{10}$

A term-model of a given theory is, in short, the model of said theory such that its domain is constructed from expressions of the object language. I will illustrate how to construct a term-model using a toy example. (The details regarding construction of a term-model are not necessary to follow the main idea of my proposal, so readers may skip the remainder of this paragraph.) Let us consider the following (very simple) theory, $P$ : $P$ consists of only two sentences ' $\exists x$ Chair $(x)$ ' and ' $\exists x \neg$ Chair $(x)$ '; the language of $P$ does not contain any constant or function symbol, and its only non-logical predicate is 'Chair'. To construct the term-model of $P$, we first extend $P$ by the following procedures: (i) for every existential formula of $P$, add its instantiation with some constant newly introduced to the language - for concreteness, suppose that 'Chair $(a)$ ' and ' $\neg$ Chair $(b)$ ' are added respectively; (ii) generate a maximally consistent theory by extending the result of (i); (iii) if the result of (ii) contains a new existential without its instantiation, add such instantiation with some newly introduced constant again, and if the theory resulting from this operation is not maximally consistent, extend the theory and make it so again. In general, the procedure (iii) can be iterated infinitely many times, but here, for simplicity, we suppose that (ii) is carried out appropriately and (iii) is vacuous. Now, let $P^{\dagger}$ be the resulting theory from (i)-(iii). We can then build the term-model $m^{\dagger}$ of $P^{\dagger}$ as follows: (1) the domain of $m^{\dagger}$ is the set consisting only of $\left\{{ }^{\prime} a^{\prime}\right\}$ and $\left\{{ }^{\prime} b '\right\}$; (2) for the valuation by $m^{\dagger},\left\{{ }^{\prime} a\right.$ ' $\}$ is assigned to ' $a$ ', $\left\{{ }^{\prime} b\right.$ ' $\}$ is assigned to ' $b$ ', and $\left\{\left\{^{\prime} a^{\prime}\right\}\right\}$ is assigned to 'Chair'. We can then easily see that $m^{\dagger}$ satisfies $P^{\dagger}$ and therefore $P$ too. More generally, the term-model for an extended theory by the above procedures can be constructed in the

${ }^{10}$ For details, see some standard textbooks of logic, e.g. van Dalen (2004), Ch.3. In developing the simplified example below, I also learned from the illustration at Williams (2007), Section 3. Originally, this method of constructing a term-model was developed by Henkin (1949) to give a proof of the completeness of classical first-order logic. 
following ways: ${ }^{11}$ (i) its domain is the set of all equivalence classes of constants, such that this equivalence relation is " $\alpha=\beta$ ' is a member of said theory"; (ii) for each constant symbol ' $\alpha$ ', the equivalence class of which ' $\alpha$ ' itself is an element is assigned; for each $n$-ary predicate symbol ' $A$ ', its assignment is the class of the ordered $n$-tuples of assignment to ' $\alpha_{1}$ ', ... ' $\alpha_{n}$ ' such that ' $A \alpha_{1} \ldots \alpha_{n}$ ' is a member of said theory.

In the same manner as illustrated, we can construct a term-model for any consistent first-order theory. Hereafter, ' $m^{t T}$ ' $\left(=\left\langle D^{t T}, V^{t T}\right\rangle\right)$ is used as the specific name for the term-model of $T$.

\subsection{Linguistic Ontology and the One as Semantic Glue}

Based on the considerations in this section thus far, I can present the package of LOOSG. Presupposing the standard framework of model-theoretical semantics, LOOSG comprises the following two theses, as well as DT, stated in 3.1:

OSG The following conditions hold for the One: (1) the One attaches the vocabularies of $m^{t T}\left(=\left\langle D^{t T}, V^{t T}\right\rangle\right)$ to the valuations by $V^{t T}$ owing to the One's special semantic force; $(2)$ for any model $m(=\langle D, V\rangle)$, if the One attaches the vocabularies of $m$ to the valuations by $V$ owing to the One's special semantic force, then $m=m^{t T}$; (3) except for the One, nothing has such special semantic force.

SGSRP For any model $m(=\langle D, V\rangle)$ of $T$, it is sufficient for $m$ 's being referentially privileged that there is an entity such that (1) it attaches the vocabularies of $m$ to the valuations by $V$ owing to its special semantic force; (2) for any model $m^{*}\left(=\left\langle D^{*}, V^{*}\right\rangle\right)$, if it attaches the vocabularies of $m^{*}$ to the valuations by $V^{*}$ owing to its special semantic force, then $m^{*}=m$; (3) except for this entity, nothing has such special semantic force.

It is straightforwardly observed that LOOSG is consistent with the claim of EM.

I will now comment on each thesis. OSG (the abbreviation of "the One as Semantic Glue") is the main thesis and is based on the idea that the One is a distinctive entity manifesting a special and unique semantic power. In other words, it is supposed that the One plays the role of what Theodore Sider describes as the semantic glue or magnet (Sider (2011), Ch.3). Furthermore, this thesis claims that the special semantic power of the One has manifestation precisely at the term-model of $T$. The One is supposed to be a unique being that has special semantic magnetism to combine the vocabularies of $T$ with mathematical constructs from the constant symbols of (extended) T. In my view, conceiving the One as such a being is reasonable, or at least, not unintelligible for an existence monist - if we endorse some kind of monistic view, the idea of attributing some special capability to the One is fairly

\footnotetext{
${ }^{11}$ For simplicity, I omit the cases in which the language contains function symbols.
} 
intelligible. ${ }^{12}$ In particular, the view that the One serves as the semantic magnet gluing the constituents of the term-model of $T$, is fairly admissible for an existence monist. (Or, in my view, there is seemingly no decisive reason for an existence monist to dismiss such a view as unacceptable.)

Let us move on to SGSRP (the abbreviation of "Semantic Glue is Sufficient for Referentially Privileged"). SGSRP is intended as a principle that provides the ground for a model to be referentially privileged. In this regard, SGSRP is expected to serve as an alternative to Lewis's eligible model approach. Nevertheless, as a conditional principle, SGSRP is seemingly acceptable by most metaphysicians. This principle reflects the following thought: if a special entity exists which possesses semantic magnetism such that vocabularies can be attached to their referents from a given model, then the existence of such an entity can serve as sufficient ground for the fact that said model is referentially privileged. This conditional claim has plausibility in a rather trivial sense. If positing an entity with such semantic magnetism is allowed, then such an entity will itself serve as the grounds for a given model's being referentially privileged. In this case, such an entity itself provides the reason by which such a model is referentially privileged. As will be unsurprising for many, the real problem with SGSRP is whether the antecedent holds or not. Most metaphysicians, other than those identifying as existence monists, have no reason or motivation to support this antecedent. Rather, these metaphysicians will be highly skeptical of the existence of an entity with a special semantic force. For such metaphysicians, the idea of eligible model would be an attractive proposal. However, even for those who embrace the eligible model approach, SGSRP as a hypothetical claim will be acceptable in a trivial sense. Thus, SGSRP should be sufficiently acceptable for an existence monist as well.

The assessment of OSG and SGSRP above suggests that both theses are reasonably acceptable to an existence monist. Once LOOSG is accepted, the explanation for truisms is obtained straightforwardly - in other words, given LOOSG, we can deduce that $T$ is actually true. From OSG, it follows that the One meets the conditions (1)-(3) of SGSRP. Thus, $m^{t T}$ is referentially privileged. Because $m^{t T}$ also satisfies $T$ by the definition of $m^{t T}$, the right-hand side of DT holds, and $T$ is therefore true. Here, the logical/inference relation among the members of $T$ is trivially preserved. Furthermore, it should also be noted that these results are obtained using the familiar framework of standard model-theoretical semantics. In other words, LOOSG has the theoretical virtue of technical simplicity - LOOSG does not need to develop a novel language exclusively talking about the One and its modes, nor does it need to deviate from the framework of standard semantics already familiar to us. Based on

${ }^{12}$ In Spinozistic monism, the One has some divine nature, and we can perhaps discuss the One's special semantic capability in relation to such a divine nature. However, considering the topics related to theological matters is beyond the scope of this paper. 
these considerations, I offer LOOSG as a viable option for existence monism.

\section{Possible Objections and Replies}

Some objections to LOOSG can be easily expected and I will reply to those in this section.

\section{Conflict with the obvious truths about interpretation?}

Objection: According to LOOSG, the vocabularies of $T$ are interpreted in a very eccentric manner. That is, those vocabularies are interpreted in such a way that the mathematical constructs from the vocabularies themselves are assigned as their semantic values. However, these eccentric assignments conflict with the obvious truths related to reference or denotation. It is intuitively or empirically obvious that, for example, 'chair' denotes the very (concrete and material) chairs. In other words, LOOSG is inconsistent with truisms related to the interpretation of our language.

Reply: Rather, we should consider that the obvious truths about reference/denotation are also included in $T$. In the package of LOOSG, such truths should also be reinterpreted by the term-model and the extent of their truth-hood can be preserved in this way. From another viewpoint, we can appeal to a style of reply in line with the "just more theory" approach here. ${ }^{13}$ We can add any sentence that seemingly states some obvious truth to the totality of truisms $T$ (as long as the consistency of $T$ is preserved). ${ }^{14}$

\section{Term-model as a referentially privileged model is absurd?}

Objection: LOOSG says that the term-model of $T$ is its referentially privileged model. However, this is the very absurd point - as stated in Section 3.1, the notion of referentially privileged model was based on that of intended model, and what is called

13 Originally the phrase "just more theory" argument' was used in realism-antirealism debate as the name for a specific reply defending the indeterminacy of reference (cf. Lewis (1984), Sider (2011), Ch.3)). Here, the use of this term is certainly outside of the original context.

${ }^{14}$ However, it should also be noted that not all truths admitted in the framework of LOOSG are included in truisms. For example, the claim of EM itself cannot be included in $T$ (adding ' $\exists x(C x \& \forall y(C y \rightarrow y=x)$ )' to $T$ will immediately make $T$ inconsistent). Here, we have to make a distinction between the truths to be explained, which belong to truisms, and the explaining truths, which belong to the semantic metatheory. $T$ is the former, and EM as well as other theses comprising LOOSG is the latter. The latter truths themselves will require some different semantics other than what LOOSG provides - perhaps it is viable to consider that LOOSG itself is stated in the fundamental language and part of metaphysical semantics as discussed in Sider (2011) (esp. Ch.7). Anyway, this is another topic and not to be discussed within the scope of this paper. 
an intended model is not a term-model in most cases. Generally speaking, an intended model of a given theory is such a model that its reference/interpretation is intuitively appropriate. In other words, the intended model of truisms $T$ must be the one at which, for example, the valuation of 'chair' is the very (concrete and material) chairs and the valuation of 'table' is the very (concrete and material) tables. And the term-model of $T$ is, of course, never the one at which such valuations are given.

Reply: As I mentioned in the footnote 9, a referentially privileged model of a given theory does not have to be its intended model. In fact, the notion of referentially privileged model was introduced by extracting the function of models usually called intended models, and this function is, as stated in Section 3.1, to determine the reference/interpretation of a given theory. ${ }^{15}$ Thus, what LOOSG actually asserts is that the reference/interpretation of $T$ is the one provided by the term-model of $T$. Of course, this claim itself is against the thought which mainstream metaphysicians have as to how the reference/interpretation is determined. However, dismissing this claim just because of its being against the mainstream thought is question-begging here. That is, what LOOSG provides is an alternative and nonstandard proposal as to which model is a referentially privileged one. And this alternative proposal should be evaluated from the viewpoint of whether the explanations which the existence monist desires are furnished given this proposal. If someone rejects the idea of the term-model as a referentially privileged model of $T$ just because this idea is eccentric, it is considered begging the question here.

\section{LOOSG remains too underspecified?}

Objection: LOOSG makes very few statements regarding the ontological status of the One except that regarding its role as a semantic glue. For example, what features or properties does the One instantiate other than being semantic glue? To these questions, LOOSG seemingly provides few answers, and the ontological nature of the

${ }^{15}$ An anonymous referee worried that a referentially privileged model without being an intended model might lose the power to define absolute truth. The referee's concern is as follows: the notion of intended model reflects the ordinary usage of reference/interpretation, and it is likely that this fact makes it plausible to define absolute truth as truth in the intended model; thus, a referentially privileged model separated from intended models may undermine the plausibility of DT.

My view on this point is basically that just having the function to determine the reference/interpretation (of a given theory) is enough to characterize absolute truth. In my view, we do not have to presuppose the ordinary usage or notion of reference/interpretation in order to conceive the notion of truth simpliciter; rather, what we need here is to just guarantee the privilege of some model(s), and the function itself to determine the reference/interpretation (of a given theory) is enough to perform this task. However, I must also admit that I am not prepared for developing a satisfactory argument over this point here. In any case, thanks to the anonymous referee for pressing me to consider this issue. 
One remains quite obscure. (This point should be contrasted with another version of existence monism, such as that which identifies the One with the whole cosmos.) Moreover, the nature of the special semantic force attributed to the One is not explained at all. This special force is just described as such, and no further explication is provided. These points suggest that LOOSG lacks any substantial metaphysical picture and raises the possible concern that LOOSG does not qualify as a specific version of existence monism.

Reply: On the whole, I must admit that LOOSG still remains underspecified to some extent. However, we may regard LOOSG as a theoretical frame within which we can develop more substantial metaphysics of the One. For example, as to the viewpoint that identifies the One with the cosmos, this viewpoint itself does not conflict with the thesis asserting that the One serves as a semantic glue. Thus we can attribute many ontological features to the One, unless a given feature prompts a conflict with the idea that the One serves as a semantic glue.

As to the nature of special semantic force, we may flesh out more detailed metaphysics as well; but it is also possible to regard the notion of special semantic force as a primitive idea. It is familiar for a contemporary metaphysician to obtain some explanatory results at the cost of a questionable ideology when she builds any metaphysical framework. (Here, I can also emphasize that the notion of special semantic force is not as extremely questionable as its first impression - after all, the notion of semantic glue, which manifests some kind of special semantic force, is embraced by more than a few contemporary metaphysicians (e.g. Sider (2011))). So, instead of explicating the notion of special semantic force unique to the One, positing this notion as a primitive seems a viable option too.

\section{OSG and SGSRP are not justified enough?}

Objection: In Section 3.3, the main theses of LOOSG (OSG and SGSRP) were just postulated and no proper justification has been given to each thesis thus far. Some expositions of the aims of each thesis were given, but those expositions only say that OSG or SGSRP are seemingly acceptable for an existence monist - that is, there is no decisive reason for the existence monist to reject OSG/SGSRP as unacceptable. And of course, that some theses are just acceptable does not mean that those are justified. Stated differently, the alleged results obtained by LOOSG just show that given the presupposition that the One possesses some convenient (and unordinary) properties, it provides the desired explanation. And the presupposition about the One is just posited in a literal sense.

Reply: In the first place, I emphasize that the aim of this paper is quite restricted: to provide an alternative and viable option for an existence monist. Thus, it is beyond the scope of this paper to provide a justification for OSG and SGSRP which will 
persuade the people who are not existence monists. Only presenting another viable option for an existence monist will serve the purpose of the paper.

Now, as observed in Section 3.3, there is no obvious reason to reject OSG/SGSRP for an existence monist. Then, the problem is as follows: for the existence monist, is there any positive reason to adopt these presuppositions? To answer this question positively, actually we do not have to give a direct justification for those theses here. We can appeal to more indirect justifications in the context of this kind of discussion; those presuppositions can be justified on the basis that the desired explanations are obtained due to positing those. As showed, LOOSG has the problem-solving power of resolving the prima facie inconsistency with truisms. This problem-solving power itself gives a motivation for OSG/SGSRP, and such motivation will provide one definite reason to adopt those presuppositions for the existence monist. Of course, this approach of indirect justification will only work for the existence monist - the problem of inconsistency with truisms is only a problem for existence monists. However, as I stated, that will be enough for this paper's purpose.

\section{The choice of $m^{t T}$ is arbitrary?}

Objection: Since it is an elementary result of metalogic that there are infinitely many $m$ s such that $m$ is isomorphic to $m^{t T}$ and $m$ 's domain is constructed from vocabularies, in fact we should also say that there are (infinitely) many term-models of $T$. Among the infinite number of term-models, why is a specific $m^{t T}$ identified as referentially privileged? Here, there is seemingly no reason to do so.

Furthermore, we can also note the existence of an infinite number of models of $T$, such that they are isomorphic to $m^{t T}$ and their domains are constructed from purely mathematical objects (for example, numbers). These "mathematical" models substantially play the same role as $m^{t T}$ in model theory. Again, why is only $m^{t T}$ qualified as privileged, while those with purely mathematical domains are not? ${ }^{16}$

Reply: To the former objection, actually we do not have to determine the unique $m^{t T}$ as referentially privileged. Most metaphysicians endorsing DT would not have a serious trouble with some mild indeterminacy of interpretation and could admit that some multiple models are equally referentially privileged. ${ }^{17}$ In addition, in the framework provided by LOOSG, it is unnecessary to think that the difference among term-models plays an important role in the idea of OSG. Thus, in my view, it is permissible for defenders of LOOSG to hold that every term-model of $T$ is qualified as referentially privileged by the One.

In response to the latter objection, my argument is as follows: in fact, we can develop a mathematical or Pythagorean version of LOOSG. According to this version,

${ }^{16}$ For details about these results, see e.g. van Dalen (2004) again.

17 cf. Williams (2007), Section 2. 
the One picks up some mathematical model of $T$ as referentially privileged. The original question then moves into the following: of the original or mathematical version, which is preferable? Here, what may provide some motivation for the original LOOSG is a kind of linguistic idealistic picture: if the original version is adopted, the truth-condition for any member of $T$ will be ultimately specified as some condition talking about constructs from linguistic objects. This likely suggests that truisms are explained at the linguistic level in one sense. Thus, if such a picture has more attractive points for a defender of LOOSG than the Pythagorean picture, she has reason to prefer the original LOOSG. Certainly, these speculations alone do not settle the problem. However, the following should be noted: (1) choosing either version does not necessarily involve arbitrariness, and (2) the problem of deciding between these versions is actually a competition between the two versions of LOOSG.

\section{Concluding Remarks}

As we have seen, LOOSG can provide an explanation for truisms by employing some elementary metalogical results and can also furnish an approach to overcome the major difficulty confronted by existence monism somewhat easily. Certainly, the necessity of elaborating or developing some points remains. Nevertheless, considering how few approaches have been proposed to defend the possibility of existence monism, LOOSG seemingly qualifies as a viable option for existence monism if one is endeavoring to defend it properly.

\section{References}

Bradley, F. H. (1994), Writings on Logic and Metaphysics. James W. Allard and Guy Stock (eds.), Oxford University Press.

Cornell, D.M. (2013), "Monism and Statespace Structure: A Reply to Sider", Analysis 73: 230-6.

Goff, P (ed.). (2012), Spinoza on Monism. Palgrave Macmillan.

Henkin, L. (1949), "The Completeness of the First-Order Functional Calculus", The Journal of Symbolic Logic 14: 159-66.

Horgan, T. \& Potrč, M. (2008), Austere Realism: Contextual Semantics Meets Minimal Ontology. MIT Press.

Korman, D. Z. (2008), "Review of Horgan and Potrč's Austere Realism" Notre Dame Philosophical Reviews, 2008,10.22, https://ndpr.nd.edu/news/austere-realism-contextualsemantics-meets-minimal-ontology/

Lewis, D. (1983), "New Work for a Theory of Universals", Australasian Journal of Philosophy 61: 343-77.

Lewis, D. (1984), "Putnam's Paradox", Australasian Journal of Philosophy 62: 221-36.

Lowe, E. J. (2012), "Against Monism", in Goff (2012), pp.92-112.

Parmenides. On Nature. In Diels, H. \& Kranze, W. (eds.) (1951-52), Die Fragmente der 
Vorsokratiker, 6th ed. Weidmann.

Schaffer, J. (2007), "From Nihilism to Monism", Australasian Journal of Philosophy 85: $175-91$.

Schaffer, J. (2008), "On What Grounds What", in Chalmers, D., Manley, D. \& Wasserman, R. (eds.) (2008), Metametaphysics. Oxford University Press, pp. 347-83.

Schaffer, J. (2012), "Why the World has Parts: Reply to Horgan \& Potrč", in Goff (2012), pp. $77-91$.

Schaffer, J. (2018), "Monism", in Zalta, E. N. (ed.), The Stanford Encyclopedia of Philosophy (Winter 2018 Edition), URL= https://plato.stanford.edu/archives/win2018/entries/monism/

Sider, T. (2007), "Against Monism", Analysis 67: 1-7.

Sider, T. (2008), "Monism and Statespace Structure", in Robin Le Poidevin (ed.) (2008), Being: Developments in Contemporary Metaphysics. Cambridge University Press, pp. $129-50$

Sider, T. (2011), Writing the Book of the World. Oxford University Press.

Spinoza, B. (1677), Ethics. In the Collected Writings of Spinoza vol.1 (1985), Princeton University Press.

van Dalen, D. (2004), Logic and Structure. 4th ed. Springer.

Williams J. R. G. (2007), "Eligibility and Inscrutability", Philosophical Review 116: 361-99.

(Received 2020.3.31; Revised 2020.8.8; Accepted 2020.8.17) 